\title{
高分辨雷达距离一多普勒扩展目标广义自适应 子空间检测器
}

\author{
戴奉周，刘宏伟*，吴顺君 \\ 西安电子科技大学雷达信号处理国家重点实验室, 西安 710068 \\ * 通信作者. E-mail: hwliu@xidian.edu.cn \\ 收稿日期: 2009-04-27; 接受日期: 2009-12-03 \\ 国家自然科学基金 (批准号: 60772140,60901068) 资助项目
}

\begin{abstract}
摘要 本文提出了一种非高斯杂波中的距离 - 多普勒扩展目标广义自适应 子空间检测器 (RDST-GASD). 采用了频率 - 慢时间子空间模型来描述宽带雷 达回波信号序列. 杂波的统计模型采用球不变随机向量描述, 各距离单元内 的杂波具有相同的归一化协方差矩阵和不同的功率 (非均匀), 并且假设可以 得到只含有杂波的参考单元的数据以估计杂波的协方差矩阵. 通过理论分 析证明了 RDST-GASD 对杂波的功率和协方差矩阵都具有恒虚警的性质.对 于宽带雷达, 在相参处理时间内, 目标回波通常存在越距离单元走动, RDST-
\end{abstract}

关键词

高分辨雷达

距离一多普勒扩展目标

距离走动

球不变随机向量

自适应子空间检测器

\section{1 引言}

高分辨雷达可以沿视线方向分辨目标上的多个散射中心, 此时目标称为距离扩展目标. 高分辨雷 达可以获得更多的目标信息, 以实现对目标的成像、分类、识别以及高精度参数测量. 在杂波环境中, 提高距离分辨率可以降低每个距离单元中杂波的后向散射功率, 从而改善雷达的检测性能 ${ }^{[1]}$. 但另一 方面, 高分辨雷达检测也存在一定困难, 主要体现在以下 3 个方面: 1) 每个距离单元内独立散射点数 目减少, 根据中心极限定理得出的高斯杂波模型不再适用;2) 目标检测模型不再是噪声和杂波中一个 点的检测问题, 而成为一个序列检测的问题; 3) 目标的越距离单元走动导致多次回波间相参积累算法 变得更加复杂.

近年来, 已有很多研究高分辨雷达目标检测问题的论文发表. 文献 $[1 \sim 3]$ 研究了基于多接收通道 的非高斯杂波中的距离扩展目标检测问题. 文献 [1] 设计分析了均匀和部分均匀杂波环境中距离扩展 目标的广义似然比检测器 (GLRT); 文献 [2] 将文献 [1] 中的工作推广到了同时存在杂波和干扰的环境 中; 文献 [3] 设计了非均匀杂波中距离扩展目标的广义似然比检测器. 文献 [4] 研究了在非均匀杂波中 基于多次相参回波的距离扩展目标检测问题. 以上文献在设计检测器时, 为简单起见都没有考虑目标 在不同接收通道间或多次回波间的距离走动. 
目标相对于雷达的运动除平动外常常还有转动分量; 另外在目标相对于雷达做整体平动的同时, 目标或目标上的某些部分往往还存在机械振动或转动, 引起所谓的微多普勒效应 ${ }^{[5]}$; 在上述两种情况 下, 目标回波的多普勒谱都会有所展宽, 对于宽带雷达, 此时目标成为距离 - 多普勒扩展目标. 文献 [6] 在目标的二维扩展函数已知的条件下设计了高斯白噪声中的双扩展目标最优检测器; 文献 [7] 设计分 析了非均匀杂波环境中距离 - 多普勒扩展目标的广义似然比检测器, 文中也忽略了相参处理时间内目 标的越距离单元走动.

实际上, 对于高分辨雷达, 目标在相参处理时间内存在的越距离单元走动通常是不可忽略的. 本 文建立了存在距离走动时的距离 - 多普勒扩展目标回波信号的距离频域子空间模型; 同时利用距离域 的非均匀杂波在距离频域成为部分均匀杂波这一性质, 设计了相应的自适应子空间检测器, 实现了在 非均匀杂波中对存在越距离单元走动的宽带雷达目标的有效检测.

\section{2 信号和杂波模型}

\section{1 距离 - 多普勒扩展目标子空间模型}

如引言中所述, 目标回波的多普勒扩展是由两类原因引起的. 如果目标相对于雷达的转速较慢, 或者目标微多普勒频率变化较慢, 则多普勒扩展可以用多个线性相位的离散多普勒频点描述, 这一近 似模型已在文献 [7] 中使用. 为了便于分析, 我们也使用了这个多普勒扩展模型.

设雷达发射具有大时宽 - 带宽积的线性调频相参脉冲串, 相参处理时间内发射了 $M$ 个脉冲. 待 检测目标分布在径向上连续 $L$ 个距离单元内, 且第一个距离单元与雷达间的初始径向距离为 $R_{0}$. 目 标相对于雷达的平动速度是 $v_{0}$, 而多普勒谱以 $v_{0}$ 对应的多普勒频率为中心扩展到 $I+1$ 个连续的多 普勒分辨单元中. 根据以上条件, 第 $m$ 次回波的复包络为

$$
\begin{aligned}
s\left(t_{k}, m T_{r}\right)= & \sum_{l=1}^{L} \sum_{i=-I / 2}^{I / 2} a(l, i) p\left[t_{k}-\frac{2\left(R_{0}+(l-1) \Delta R+\left(v_{0}+i \Delta v\right)\left(t_{k}+m T_{r}\right)\right)}{\mathrm{c}}\right] \\
& \times \exp \left[-\frac{j 4 \pi f_{c}}{\mathrm{c}}\left(R_{0}+(l-1) \Delta R\right)+\left(v_{0}+i \Delta v\right)\left(t_{k}+m T_{r}\right)\right]
\end{aligned}
$$

式中 $p\left(t_{k}\right)$ 是发射信号的复包络, $f_{c}$ 是雷达载频, $T_{r}$ 是脉冲重复周期, $\Delta R$ 是一个距离分辨单元的长 度, $\Delta v$ 是一个多普勒分辨单元对应的速度增量, 即 $\Delta v=\mathrm{c} /\left(2 T_{r} M f_{c}\right), a(l, i)$ 是第 $(l, i)$ 个距离 - 多普 勒分辨单元中散射点的后向散射幅度, $\mathrm{c}$ 是光速. (1) 式给出的信号模型隐含了在相参处理时间内目标 没有发生横向越距离单元走动的假设.

回波包络中的延时项 $2 v_{0} m T_{r} / \mathrm{c}$ 会引起越距离单元走动, 并且该项难以在距离域直接处理, 因此 我们通过 DFT 将回波信号变换到距离频域, 并在距离频域进行匹配滤波, 其结果是

$$
\begin{aligned}
S\left(f, m T_{r}\right)= & P\left(f-f_{d_{i}}\right) P^{*}(f) \sum_{l=1}^{L} \sum_{i=-I / 2}^{I / 2} A(l, i) \exp \left[-\frac{\mathrm{j} 4 \pi}{\mathrm{c}}\left(f+f_{c}\right)\left(v_{0}+i \Delta v\right) m T_{r}\right] \\
& \times \exp \left[-\mathrm{j} \frac{\mathrm{j} 4 \pi f}{\mathrm{c}}\left(R_{0}+(l-1) \Delta R\right)\right],
\end{aligned}
$$

式中 $P(f)$ 是发射信号的频谱, $f_{d_{i}}=2\left(v_{0}+i \Delta v\right) f_{c} / \mathrm{c}$ 是第 $i$ 个多普勒单元的多普勒频率, $A(l, i)$ 是第 $(l, i)$ 个距离 - 多普勒单元的后向散射幅度和初始相位的组合, 即 $A(l, i)=a(l, i) \exp \left[-\mathrm{j} 4 \pi f_{c}\left(R_{0}+(l\right.\right.$ $-1) \Delta R) / c]$. 
因为多普勒频率是远小于信号频率的, 所以 $P\left(f-f_{d_{i}}\right) P^{*}(f) \approx|P(f)|^{2}$. 而线性调频信号的能量 谱近似为一个宽度是带宽的矩形, 因此脉压后每个距离单元的回波包络都是一个 sinc 函数. 设采样率 等于发射信号带宽, 则采样后只需考虑每个距离单元的主瓣. 假设检测窗长是 $N$, 那么脉压后回波信 号的离散谱为

$$
\begin{aligned}
S(n, m)= & \sum_{i=-I / 2}^{I / 2} \exp \left(-\frac{j 4 \pi n}{N c} f_{s} R_{0}\right)\left[\sum_{l=1}^{L} A(l, i) \exp \left(-\frac{\mathrm{j} 4 \pi n}{N c} f_{s}(l-1) \Delta R\right)\right] \\
& \times \exp \left[-\frac{\mathrm{j} 4 \pi}{\mathrm{c}}\left(\frac{n}{N} f_{s}+f_{c}\right)\left(v_{0}+i \Delta v\right) m T_{r}\right],
\end{aligned}
$$

式中 $n$ 是离散频域变量, 取值从 0 至 $N-1 . S(n, m)$ 的第二个指数项表明目标回波的多普勒在信号 带宽范围内随信号频率变化, 称为 “多普勒色散”, 它对应于目标回波在距离域的距离走动.

根据 $S(n, m)$ 可以建立距离 - 多普勒扩展目标的距离频域子空间模型:

$$
\boldsymbol{S}_{n}=\boldsymbol{P}_{n} \boldsymbol{b}_{n}
$$

其中 $\boldsymbol{P}_{n}$ 是一个 $M \times(I+1)$ 维的矩阵, 称为导向矩阵, 它的列空间就是第 $n$ 个频点上的信号子空间,

$$
\boldsymbol{P}_{n}=\left(\begin{array}{cccc}
1 & 1 & \ldots & 1 \\
\exp \left[-\mathrm{j} \phi_{n,-I / 2}(1)\right] & \exp \left[-\mathrm{j} \phi_{n,-I / 2+1}(1)\right] & \ldots & \exp \left[-\mathrm{j} \phi_{n, I / 2}(1)\right] \\
\vdots & \vdots & & \vdots \\
\exp \left[-\mathrm{j} \phi_{n,-I / 2}(M-1)\right] & \exp \left[-\mathrm{j} \phi_{n,-I / 2+1}(M-1)\right] & \ldots & \exp \left[-\mathrm{j} \phi_{n, I / 2}(M-1)\right]
\end{array}\right)
$$

式中

$$
\begin{aligned}
& \phi_{n, i}=2 \pi\left(f_{d_{n, 0}}+i \Delta f_{d_{n}}\right) m, \\
& f_{d_{n, 0}}=\frac{2 v_{0}}{\mathrm{c}}\left(\frac{n f_{s}}{N}+f_{c}\right) T_{r}, \\
& \Delta f_{d_{n}}=\frac{2 \Delta v}{\mathrm{c}}\left(\frac{n f_{s}}{N}+f_{c}\right) T_{r} .
\end{aligned}
$$

而 $\boldsymbol{b}_{n}$ 是一个 $(I+1)$ 维的列向量, 确定了回波在信号子空间中的位置,

$$
\boldsymbol{b}_{n}=\left(b_{-I / 2}(n) \cdots b_{0}(n) \cdots b_{I / 2}(n)\right)^{\mathrm{T}},
$$

式中上标 ‘ $\mathrm{T}$ ' 表示向量转置. $b_{i}(n)$ 是 $S(n, m)$ 中与脉间时间无关各项的组合,

$$
b_{i}(n)=\exp \left(-\frac{\mathrm{j} 4 \pi n}{N \mathrm{c}} f_{s} R_{0}\right)\left[\sum_{l=1}^{L} A(l, i) \exp \left(-\frac{\mathrm{j} 4 \pi n}{N \mathrm{c}} f_{s}(l-1) \Delta R\right)\right] .
$$

\section{2 高分辨雷达杂波模型}

球不变随机向量 (SIRV) 常用来描述高分辨雷达杂波 ${ }^{[8]}$. 该模型假设不同距离单元的杂波统计独 立, 而一个距离单元中连续 $M$ 次回波中的杂波用一个随机向量 $\boldsymbol{d}_{l}$ 描述, 并且 $\boldsymbol{d}_{l}=\sqrt{u_{l}} \boldsymbol{g}_{l}$, 其中 $u_{l}$ 是 一个非负随机变量, 用来描述第 $l$ 个距离单元的杂波功率, $\boldsymbol{g}_{l}$ 是一个具有单位协方差矩阵的 0 均值复 
高斯随机向量, $E\left[\boldsymbol{g}_{l} \boldsymbol{g}_{l}^{\mathrm{H}}\right]=\boldsymbol{R}\left(\boldsymbol{R}\right.$ 对角线上的元素等于 1), 并且它与 $u_{l}$ 统计独立. 式中上标 ' $\mathrm{H}$ ' 表示共 轭转置. 在该模型中, 所有距离单元杂波的归一化协方差矩阵都相同, 但是杂波功率却在距离单元间变 化, 这就是所谓的非均匀杂波环境 ${ }^{[4]}$.

以上模型在距离域描述杂波特性, 但是信号模型是在距离频域建立的, 因次我们需要分析杂波在 距离频域的统计特性. 对 $d(l, m)$ 沿 $l$ 做 DFT, 得到距离频域的杂波 $c(n, m)$. 可以证明距离频域的杂 波向量 $\boldsymbol{c}_{n}(m)$ 具有以下统计特征:

$$
\boldsymbol{c}_{n}=\sqrt{\varepsilon} \boldsymbol{k}_{n}, \varepsilon=\frac{1}{L} \sum_{l=1}^{L} u_{l}, E\left[\boldsymbol{k}_{n} \boldsymbol{k}_{n}^{\mathrm{H}}\right]=E\left[\boldsymbol{g}_{l} \boldsymbol{g}_{l}^{\mathrm{H}}\right]=\boldsymbol{R} .
$$

这表明在距离频域所有单元杂波的归一化协方差矩阵仍然相等, 并且与距离域的相同; 而所有单元杂 波的功率都等于变换前检测窗内的平均杂波功率. 这样, 距离域的非均匀杂波在距离频域变成了均匀 杂波.

\section{3 距离 - 多普勒扩展目标广义自适应子空间检测器设计}

\section{1 多普勒导向矩阵已知时的广义似然比检测器}

根据上一节中给出的距离频域的信号和杂波模型, 非均匀杂波中的距离 - 多普勒扩展目标的检测 可以用以下二元假设来描述

$$
\begin{cases}H_{0}: \boldsymbol{z}_{n}=\boldsymbol{c}_{n}, & n=0, \ldots, N-1, \\ H_{1}: \boldsymbol{z}_{n}=\boldsymbol{P}_{n} \boldsymbol{b}_{n}+\boldsymbol{c}_{n}, & n=0, \ldots, N-1 .\end{cases}
$$

该检测问题在 Neyman-Pearson 准则下的的最优解是似然比检测器. 由于不同频带内的杂波近拟相互 独立, 因此检测单元数据在 $H_{0}$ 和 $H_{1}$ 假设下的联合概率密度函数分别是

$$
f_{\boldsymbol{z}_{0}, \ldots, \boldsymbol{z}_{N-1}}\left(\boldsymbol{z}_{0}, \ldots, \boldsymbol{z}_{N-1} \mid \varepsilon \boldsymbol{R}, H_{0}\right)=\prod_{n=0}^{N-1} \frac{1}{\pi^{M} \varepsilon^{M} \operatorname{det}(\boldsymbol{R})} \exp \left(-\frac{\boldsymbol{z}_{n}^{H} \boldsymbol{R}^{-1} \boldsymbol{z}_{n}}{\varepsilon}\right)
$$

和

$$
f_{\boldsymbol{z}_{0}, \ldots, \boldsymbol{z}_{N-1}}\left(\boldsymbol{z}_{0}, \ldots, \boldsymbol{z}_{N-1} \mid \varepsilon \boldsymbol{R}, H_{1}\right)=\prod_{n=0}^{N-1} \frac{1}{\pi^{M} \varepsilon^{M} \operatorname{det}(\boldsymbol{R})} \exp \left[-\frac{\left(\boldsymbol{z}_{n}-\boldsymbol{P}_{n} \boldsymbol{b}_{n}\right)^{H} \boldsymbol{R}^{-1}\left(\boldsymbol{z}_{n}-\boldsymbol{P}_{n} \boldsymbol{b}_{n}\right)}{\varepsilon}\right],
$$

式中 $\operatorname{det}(\boldsymbol{R})$ 是 $\boldsymbol{R}$ 的行列式. 这两个联合概率密度函数中都存在多个未知参数, 例如 $\varepsilon, v_{0}, \boldsymbol{b}_{n}$ 和 $\boldsymbol{R}$ 等, 因此我们必须采用广义似然比检测, 即

$$
\frac{\max _{\varepsilon, v_{0}, \boldsymbol{b}_{0}, \ldots, \boldsymbol{b}_{N-1}} f_{\boldsymbol{z}_{0}, \ldots, \boldsymbol{z}_{N-1}}\left(\boldsymbol{z}_{0}, \ldots, \boldsymbol{z}_{N-1} \mid v_{0}, \boldsymbol{b}_{0}, \ldots, \boldsymbol{b}_{N-1}, \varepsilon \boldsymbol{R}, H_{1}\right)}{\max _{\varepsilon} f_{\boldsymbol{z}_{0}, \ldots, \boldsymbol{z}_{N-1}}\left(\boldsymbol{z}_{0}, \ldots, \boldsymbol{z}_{N-1} \mid \varepsilon \boldsymbol{R}\right), H_{0}} \underset{H_{0}}{\stackrel{H_{1}}{\gtrless}} \gamma,
$$

式中 $\gamma$ 是检测门限. 首先假设杂波的归一化协方差矩阵以及各距离频率单元的导向矩阵已知, 并求得 其余未知参数的极大似然估计. 我们可以得到 $\varepsilon$ 在 $H_{0}$ 和 $H_{1}$ 下的极大似然估计分别是

$$
\hat{\varepsilon}_{H_{0}}=\frac{\sum_{n=0}^{N-1} \boldsymbol{z}_{n}^{\mathrm{H}} \boldsymbol{R}^{-1} \boldsymbol{z}_{n}}{M N}
$$


和

$$
\hat{\varepsilon}_{H_{1}}=\frac{\sum_{n=0}^{N-1}\left(\boldsymbol{z}_{n}-\boldsymbol{P}_{n} \boldsymbol{b}_{n}\right)^{\mathrm{H}} \boldsymbol{R}^{-1}\left(\boldsymbol{z}_{n}-\boldsymbol{P}_{n} \boldsymbol{b}_{n}\right)}{M N},
$$

$\boldsymbol{b}_{n}$ 的极大似然估计是

$$
\hat{\boldsymbol{b}}_{n}=\left(\boldsymbol{P}_{n}^{\mathrm{H}} \boldsymbol{R}^{-1} \boldsymbol{P}_{n}\right)^{-1}\left(\boldsymbol{P}_{n}^{\mathrm{H}} \boldsymbol{R}^{-1} \boldsymbol{z}_{n}\right) .
$$

假设除了检测单元还可得到用于估计杂波协防差矩阵的参考单元数据. 参考单元中仅含杂波而没 有目标回波. 在距离频域, 参考单元和检测单元具有相同的归一化杂波协方差矩阵, 但是杂波的功率却 不同, 这就是所谓的部分均匀杂波环境 ${ }^{[1]}$. 不失一般性, 我们假设参考单元中杂波的功率是 1 , 这样由 参考单元得到采样协方差矩阵就是对杂波归一化协方差矩阵的估计. 为了使检测器在 $H_{0}$ 假设下的分 布与多普勒导向矩阵无关, 需要对不同的距离频率单元使用统计独立的采样协方差矩阵. 假设每个采 样协方差矩阵是由 $H M$ 个参考单元数据得到的, 则第 $n$ 个频率单元所用的采样协方差矩阵是

$$
\hat{\boldsymbol{R}}_{n}=\frac{1}{H M} \sum_{t=H M n}^{H M(n+1)-1} \boldsymbol{z}_{t} \boldsymbol{z}_{t}^{\mathrm{H}} .
$$

将这些未知参数的极大似然估计代入到 (14) 式中, 就可得到 (11) 式给出的检测问题的广义似然比检 测器

$$
T_{\mathrm{GLR}}=\frac{\sum_{n=0}^{N-1}\left[\left(\boldsymbol{P}_{n}^{\mathrm{H}} \hat{\boldsymbol{R}}_{n}^{-1} \boldsymbol{z}_{n}\right)^{\mathrm{H}}\left(\boldsymbol{P}_{n}^{\mathrm{H}} \hat{\boldsymbol{R}}_{n}^{-1} \boldsymbol{P}_{n}\right)^{-1}\left(\boldsymbol{P}_{n}^{\mathrm{H}} \hat{\boldsymbol{R}}_{n}^{-1} \boldsymbol{z}_{n}\right)\right]}{\sum_{n=0}^{N-1} \boldsymbol{z}_{n}^{\mathrm{H}} \hat{\boldsymbol{R}}_{n}^{-1} \boldsymbol{z}_{n}} .
$$

可以看出, $T_{\mathrm{GLR}}$ 的本质是检测单元数据经过杂波白化后在信号子空间中投影的功率和. $T_{\mathrm{GLR}}$ 是 一种自适应子空间检测器 ${ }^{[9]}$. 因此我们把它称为距离 - 多普勒扩展目标广义自适应子空间检测器, 为 了表示方便, 将它简称为 RDST-GASD (generalized adaptive subspace detector for range-Doppler spread target). 关于 $T_{\mathrm{GLR}}$ 还有一点需要指出, 虽然在设计检测器时我们假设参考单元中的杂波功率是 1 , 但 是这个假设并不影响结果, 因为自适应子空间检测器对于采样协方差矩阵的实常数因子具有不变性.

\section{2 多普勒导向矩阵未知时的检测方法}

在上一小节中, 我们在目标的多普勒导向矩阵已知的假设下设计了广义似然比检测器. 但实际上 目标的速度通常也是一个需要估计的量. 一种针对此类问题的基于 FFT 的检测框架在文献 [10] 中给 出. 对于该检测框架，目标速度的极大似然估计是通过搜索回波的多普勒谱峰获得的，同时将多普勒 谱的峰值与门限进行比较, 做出检测判决. 但是对于宽带雷达, 目标回波存在不可忽略的多普勒色散, 因此这种方法是不能直接使用的. 在此, 我们将基于 chirp-z 变换 (CZT) 给出一种适用于距离走动不 可忽略的宽带雷达目标的检测方法.

由于杂波是广义平稳的, 因此其归一化协方差矩阵 $\boldsymbol{R}$ 是一个 Toeplitz 矩阵. 当参考单元的数据 足够多时, 采样协方差矩阵 $\hat{\boldsymbol{R}}_{n}$ 也近似为一个 Toeplitz 矩阵. 根据 Toeplitz 矩阵的渐进性质, 当其维 数 $M$ 足够大时, 它的特征值组成的矩阵趋近于 IDFT 矩阵, 而其特征值趋近于杂波的功率谱密度, 即 $\hat{\boldsymbol{R}}_{n} \approx \boldsymbol{F} \hat{\boldsymbol{\Lambda}} \boldsymbol{F}^{\mathrm{H}}$, 其中 $\boldsymbol{F}$ 是 IDFT 矩阵. 实际上, 当矩阵维数 $M$ 大于等于 10 时, 该渐进性质有效 [11].

令 $\boldsymbol{y}_{n}=\hat{\boldsymbol{R}}_{n}^{-1} \boldsymbol{z}_{n}, \lambda_{n i}^{2}=P_{c}^{-1}\left(\hat{f}_{d_{n 0}}+i \Delta f_{d_{n}}\right)$, 那么检测器 $T_{\mathrm{GLR}}$ 可以重新表示为

$$
T_{\mathrm{GLR}}=\frac{\sum_{i=-I / 2}^{I / 2} \sum_{n=0}^{N-1} \lambda_{n i}^{2}\left|\sum_{m=0}^{M-1} \exp \left[-\mathrm{j} 2 \pi\left(\hat{f}_{d_{n 0}}+i \Delta f_{d_{n}}\right) m\right] \boldsymbol{y}_{n}(m)\right|^{2}}{\sum_{n=0}^{N-1} \boldsymbol{z}_{n}^{\mathrm{H}} \hat{\boldsymbol{R}}_{n}^{-1} \boldsymbol{z}_{n}} .
$$


如果目标在一个 CPI 内的距离走动可以忽略, 换言之, 所有频率单元的多普勒都相同, 那么 $T_{\mathrm{GLR}}$ 可以通过文献 [10] 中给出的基于 FFT 的框架来实现. 然而, 对于宽带雷达, 距离走动通常是不可忽略 的, 因此检测器的实现将比较复杂.

根据 (7) 式中给出的线性关系, 我们可以用线性调频 Z 变换 $(\mathrm{CZT})^{[12]}$ 的方法进行多普勒频率变 标, 也就是使得所有的距离频率单元具有相同的多普勒频率. 首先, 我们引入以下矩阵

$$
\boldsymbol{C}_{n}=\frac{1}{\sqrt{M}}\left(\begin{array}{cccc}
W^{00} & W^{01} & \ldots & W^{0(M-1)} \\
W^{10} & W^{11} & \ldots & W^{1(M-1)} \\
\vdots & \vdots & & \vdots \\
W^{(M-1) 0} & W^{(M-1) 1} & \ldots & W^{(M-1)(M-1)}
\end{array}\right),
$$

其中 $W=\exp \left(-\mathrm{j} 2 \pi \frac{f_{c}+n f_{s} / N}{f_{c}}\right)$.

容易看出, 第 $n$ 个距离频率单元 Doppler 导向矩阵的估计 $\hat{\boldsymbol{P}}_{n}$ 和第 0 号距离频率单元中的 Doppler 导向矩阵有如下关系

$$
\hat{\boldsymbol{P}}_{n} \approx \boldsymbol{C}_{n}^{\mathrm{H}} \boldsymbol{F} \hat{\boldsymbol{P}}_{0}
$$

将 (22) 式代入到 (19) 式中, 检测器变为

$$
T_{\mathrm{GLR}}=\frac{\sum_{n=0}^{N-1}\left[\left(\hat{\boldsymbol{P}}_{0}^{\mathrm{H}} \boldsymbol{F}^{\mathrm{H}} \boldsymbol{C}_{n} \boldsymbol{y}_{n}\right)^{\mathrm{H}}\left(\hat{\boldsymbol{P}}_{0}^{\mathrm{H}} \boldsymbol{F}^{\mathrm{H}} \boldsymbol{C}_{n} \hat{\boldsymbol{R}}_{n}^{-1} \boldsymbol{C}_{n}^{\mathrm{H}} \boldsymbol{F} \hat{\boldsymbol{P}}_{0}\right)^{-1}\left(\hat{\boldsymbol{P}}_{0}^{\mathrm{H}} \boldsymbol{F}^{\mathrm{H}} \boldsymbol{C}_{n} \boldsymbol{y}_{n}\right)\right]}{\sum_{n=0}^{N-1} \boldsymbol{z}_{n}^{\mathrm{H}} \hat{\boldsymbol{R}}_{n}^{-1} \boldsymbol{z}_{n}} .
$$

在多普勒频率变标后, 所有距离频率单元的多普勒频率都相同, 因此这一过程称之为 “多普勒聚焦”. 多普勒聚焦后的检测器 $T_{\mathrm{GLR}}$ 可以通过搜索下式的峰值来实现:

$$
T_{\mathrm{GLR}}(k)=\frac{\sum_{i=k-I / 2}^{i=k+I / 2} J(i)}{\sum_{n=0}^{N-1} \boldsymbol{z}_{n}^{\mathrm{H}} \hat{\boldsymbol{R}}_{n}^{-1} \boldsymbol{z}_{n}}, k=0,1, \ldots, M-1,
$$

式中

$$
J(k)=\sum_{n=0}^{N-1} \kappa_{n, k}^{2}\left|\sum_{m=0}^{M-1} \exp \left[-\mathrm{j} 2 \pi\left(\frac{f_{c}+n f_{s} / N}{f_{c}}\right) \frac{k m}{M}\right] y_{n}(m)\right|^{2}, k=0,1, \ldots, M-1,
$$

$1 / \kappa_{n, k}^{2}$ 是 $\boldsymbol{C}_{n} \hat{\boldsymbol{R}}_{n}^{-1} \boldsymbol{C}_{n}^{\mathrm{H}}$ 的对角线上的第 $k$ 个元素. $T_{\mathrm{GLR}}(k)$ 的峰值就是目标速度未知时的自适应检测 器. 以上提出的 RDST-GASD 实现方法的框图在图 1 中给出.

在以上的推导中, 我们都假设多普勒频率是不模糊的, 但实际情况并非总是如此. 当多普勒模糊 存在时, 我们假设模糊的次数已知, 则 (22) 式可以修正为 $\boldsymbol{P}_{n} \approx \boldsymbol{A}_{n} \boldsymbol{C}_{n}^{\mathrm{H}} \boldsymbol{F} \boldsymbol{P}_{0}$, 其中 $\boldsymbol{A}_{n}$ 是一个对角矩 阵, 对角线上的元素依次是 $W=\exp \left(-\mathrm{j} 2 \pi \frac{f_{c}+n f_{s} / N}{f_{c}}\right), m=0, \ldots, M-1$. 这样在多普勒模糊存在的时 候, 也可以利用广义自适应子空间检测器进行目标检测.

\section{4 距离多普勒扩展目标广义自适应子空间检测器的性能分析}

\section{1 距离多普勒扩展目标广义自适应子空间检测器的统计分解}

首先我们假设多普勒导向矩阵已知, 那么 RDST-GASD 可以被分解为

$$
T_{\mathrm{SD}}=\frac{\sum_{n=0}^{N-1} \frac{1}{\vartheta_{n}^{2}}\left|\boldsymbol{\alpha}_{n}+\frac{\rho_{n}}{\xi_{n}} \boldsymbol{\beta}_{n}\right|^{2}}{\sum_{n=0}^{N-1} \frac{1}{\vartheta_{n}^{2}}\left|\boldsymbol{\alpha}_{n}+\frac{\rho_{n}}{\xi_{n}} \boldsymbol{\beta}_{n}\right|^{2}+\sum_{n=0}^{N-1} \frac{\rho_{n}^{2}}{\xi_{n}^{2}}} \underset{H_{0}}{H_{1}} \gamma,
$$




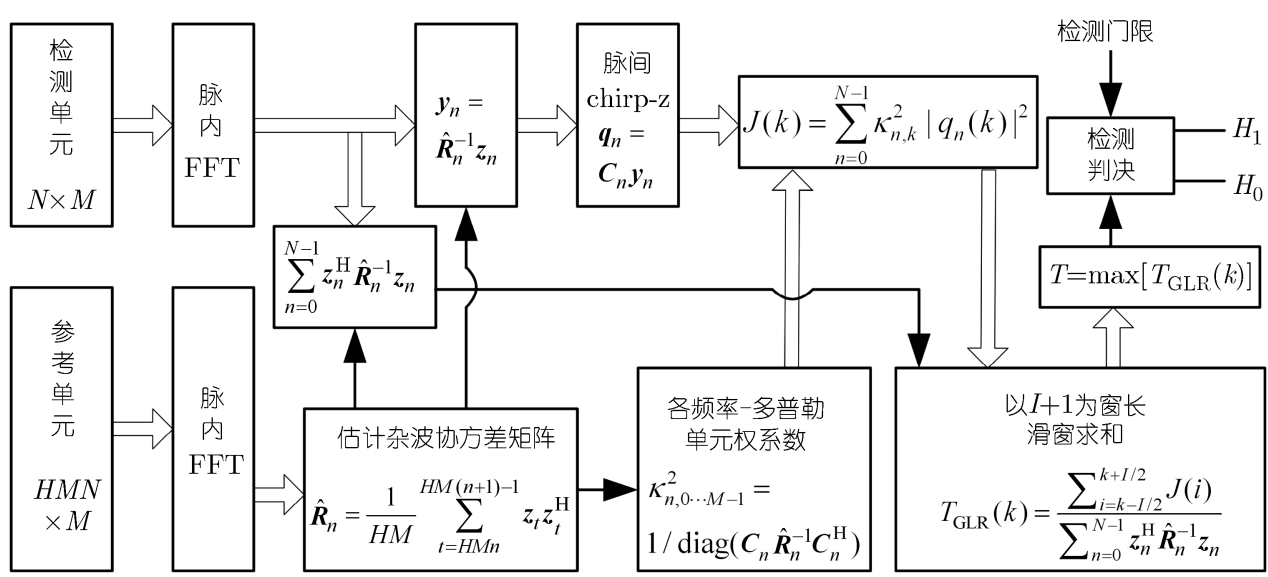

图 1 RDST-GASD 框图

其中 $\vartheta_{n}, \rho_{n}$ 和 $\xi_{n}$ 是随机变量; $\boldsymbol{\alpha}_{n}$ 和 $\boldsymbol{\beta}_{n}$ 是随机向量. 它们彼此统计独立, 并且服从的分布分别是: $\vartheta_{n}^{2} \sim$ $\Gamma(H N-M+1), \rho_{n}^{2} \sim \Gamma(M-I-1), \xi_{n}^{2} \sim \Gamma(H N-M+I+2), \boldsymbol{\alpha}_{n} \sim C N\left[\frac{1}{\sqrt{\varepsilon}}\left(\boldsymbol{P}_{n}^{\mathrm{H}} \boldsymbol{R}^{-1} \boldsymbol{P}_{n}\right)^{-1 / 2} \boldsymbol{P}_{n} \boldsymbol{b}_{n}, \boldsymbol{E}_{I+1}\right]$ 和 $\boldsymbol{\beta}_{n} \sim C N\left(0, \boldsymbol{E}_{I+1}\right)$, 其中 $\Gamma(N)$ 表示自由度为 $N$ 的 Gamma 分布, $C N(\boldsymbol{\mu}, \boldsymbol{R})$ 表示均值向量是 $\boldsymbol{\mu}$, 协方差矩阵是 $\boldsymbol{R}$ 的多维复高斯分布, $\boldsymbol{E}_{I+1}$ 表示 $I+1$ 维的单位阵. 统计分解所采用的方法与文献 [9] 相同, 在此我们省略详细过程.

(25) 式可以重新表示为

$$
\sum_{n=0}^{N-1} \frac{1}{\vartheta_{n}^{2}}\left|\boldsymbol{\alpha}_{n}+\frac{\rho_{n}}{\xi_{n}} \boldsymbol{\beta}_{n}\right|^{2} \underset{H_{0}}{\stackrel{H_{1}}{\gtrless}} \frac{\gamma}{1-\gamma} \sum_{n=0}^{N-1} \frac{\rho_{n}^{2}}{\xi_{n}^{2}} .
$$

令 $\iota_{n}=1 /\left(1+\rho_{n}^{2} / \xi_{n}^{2}\right)$, 以及 $\delta_{n}=\frac{1}{\vartheta_{n}^{2}}\left|\boldsymbol{\alpha}_{n}+\frac{\rho_{n}}{\xi_{n}} \boldsymbol{\beta}_{n}\right|^{2}$, 那么 $\iota_{n}$ 服从 $\beta$ 分布, 即 $\iota_{n} \sim \beta(H N-M+I+2, M-$ $I-1)$; 而在 $\iota_{n}$ 确定的条件下 $\delta_{n}$ 服从 $F$ 分布, 即 $\delta_{n} \mid \iota_{n} \sim F\left(I+1, H N-M+1, \boldsymbol{b}_{n}^{\mathrm{H}}\left(\varepsilon \boldsymbol{P}_{n}^{\mathrm{H}} \boldsymbol{R}^{-1} \boldsymbol{P}_{n}\right)^{-1} \boldsymbol{b}_{n}\right)$. 这样, (26) 式左边部分的条件均值是

$$
E\left(\sum_{n=0}^{N-1} \delta_{n} \mid \iota_{0}, \ldots, \iota_{N-1}\right)=\frac{1}{\varepsilon} \sum_{n=0}^{N-1} \boldsymbol{b}_{n}^{\mathrm{H}}\left(\boldsymbol{P}_{n}^{\mathrm{H}} \boldsymbol{R}^{-1} \boldsymbol{P}_{n}\right)^{-1} \boldsymbol{b}_{n} .
$$

根据以上分析, 在 $H_{1}$ 假设下 RDST-GASD 的检测概率随 (27) 式给出的条件均值而增加, 因此可以定 义信杂比为

$$
\mathrm{SCR}=\frac{1}{\varepsilon M N} \sum_{n=0}^{N-1} \boldsymbol{b}_{n}^{\mathrm{H}}\left(\boldsymbol{P}_{n}^{\mathrm{H}} \boldsymbol{R}^{-1} \boldsymbol{P}_{n}\right)^{-1} \boldsymbol{b}_{n} .
$$

在 $H_{0}$ 假设下, $\boldsymbol{b}_{n}$ 是一个 $\mathbf{0}$ 矢量, RDST-GASD 的虚警概率仅与 $H, N, M, I$, 和 $\gamma$ 有关, 而不依赖于 杂波的功率或协方差矩阵. 因此我们可以得出结论, RDST-GASD 对杂波的功率和协方差矩阵都具有 恒虚警的性质.

目标速度未知时, 估计多普勒导向矩阵的搜索过程会增加 RDST-GASD 的虚警概率, 但是不会改 变检测器的恒虚警性质. 在此忽略这部分细节, 类似的详细分析可以在文献 [13] 中找到.

\section{2 仿真结果}

本小节给出一组 Monte Carlo 仿真实验的结果, 以验证 RDST-GASD 的检测性能. 假设虚警概率 是 $10^{-4}$. 检测门限和检测概率分别通过 $10^{6}$ 和 $10^{4}$ 次独立实验来估计. 假设一个相参处理时间内有 32 
表 1 多普勒谱

\begin{tabular}{cccccc}
\hline 归一化多普勒频率 & $21 / 32$ & $22 / 32$ & $23 / 32$ & $24 / 32$ & $25 / 32$ \\
\hline 功率 & 0.18 & 0.09 & 0.46 & 0.13 & 0.16 \\
\hline
\end{tabular}

个相参脉冲. 目标扩展到 56 个距离单元中, 并且在相参处理时间内越过了 8 个距离单元, 检测窗的长 度是 64 个距离单元. 目标的多普勒谱扩展到 5 个多普勒单元中, 在表 1 中给出.

仿真实验 1: 为了验证 RDST-GASD 的有效性, 以及在 4.1 小节中对其进行统计分析的正确性, 在 图 2 中我们画出了 (25) 式给出的经过统计分解后的检测统计量 $T_{\mathrm{SD}}$ 与 RDST-GASD 的检测性能曲 线. 设参考单元的长度是检测单元长度的 8 倍, 即 $H=8$.

从图 2 中可以看出, 当用 (24) 式实现 RDST-GASD 时, 其检测性能比式 (25) 给出的理论分析得 到的检测性能要差一些, 在检测概率等于 0.9 处大约下降 $1 \mathrm{~dB}$. 这个原因是多方面的, 其中最主要因 素是 $T_{\mathrm{SD}}$ 是在目标速度已知的条件下做统计分解得到的检测统计量, 而在 (24) 式中是通过搜索来完 成对目标速度的估计的，而搜索过程会引起虚警概率的增加，为了保证恒虚警必须提高门限，从而检 测性能也就会随之下降. 另外对杂波协方差矩阵特征值分解性质的近似以及式 (22) 给出的多普勒频 率变标的近似等式也都会引起 RDST-GASD 性能的下降. 该实验结果可以证明本文给出的检测器对 于杂波中的宽带距离多普勒扩展目标的检测是有效的, 对其统计性质的分析也是正确的.

仿真实验 2: 对于距离 - 多普勒扩展目标, 忽略目标在多普勒上的扩展将会引起检测性能的下降. 例如, 对于表 1 给出的信号多普勒谱模型, 如果目标在多普勒上的扩展被忽略, 在通过 (24) 式计算 RDST-GASD 时, 多普勒谱窗的长度取值为 1 , 此时的检测器对于只存在距离扩展的目标是适用的, 记 为 RST-GASD. 设 $H=8$. 图 3 画出了两个检测器的检测性能曲线. 由于 RDST-GASD 沿多普勒频率 对目标多普勒谱进行能量积累, 因此 RDST-GASD 较 RST-GASD 的检测性能有明显改善, 在检测概 率等于 0.9 时 RDST-GASD 的检测性能比 RST-GASD 提高约 $2 \mathrm{~dB}$.

仿真实验 3: 分析参考单元长度对 RDST-GASD 检测性能的影响.

图 4 画出了在杂波协方差矩阵 $\boldsymbol{R}$ 已知 (用 $H=\infty$ 表示) 和参考单元长度与检测单元长度之比分 别为 2,4 和 8 时 RDST-GASD 检测性能曲线. RDST-GASD 的性能损失随着的参考单元个数的下降 而增加, 当 $H=2$ 时, 相比于 $\boldsymbol{R}$ 已知时的性能损失约为 $3 \mathrm{~dB}$.

仿真实验 4: 分析多普勒聚焦对 RDST-GASD 检测器的性能改善. 设 $H=8$.

如前文中分析, 距离频域的多普勒聚焦等价于距离域的目标回波距离走动校正, 因此做多普勒聚 焦后 RDST-GASD 对目标回波做了相参积累, 而不做多普勒聚焦只相当于对目标回波做了非向参积 累, 将未做多普勒聚焦的检测器记为 NDF-GASD. 从图 5 可以看出, 如果不做多普勒聚焦, NDF-GASD 相对于 RDST-GASD 的性能损失在检测概率为 0.9 时达 $6 \mathrm{~dB}$ 以上, 这基本符合回波次数等于 32 时 非相参积累检测器相对于相参积累检测器的性能损失.

仿真实验 5: RDST-GASD 在不同杂波参数下的检测性能比较. 设 $H=8$.

以上几组仿真实验中都假设在相参处理时间内杂波的功率保持不变, 但实际上正如本文的第 2 部 分所分析的, 杂波的包络用球不变随机向量模型的慢变分量表示, 是具有一定分布的非负随机变量, 此 时 (28) 式定义的信杂比应修正为

$$
\mathrm{SCR}=\frac{1}{E(\varepsilon) M N} \sum_{n=0}^{N-1} \boldsymbol{b}_{n}^{\mathrm{H}}\left(\boldsymbol{P}_{n}^{\mathrm{H}} \boldsymbol{R}^{-1} \boldsymbol{P}_{n}\right)^{-1} \boldsymbol{b}_{n} .
$$




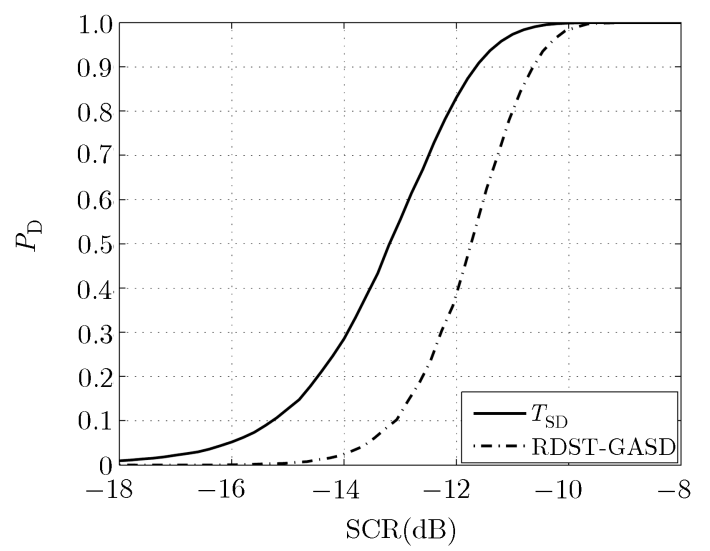

图 2 RDST-GASD 相对于 $T_{\mathrm{SD}}$ 的性能损失

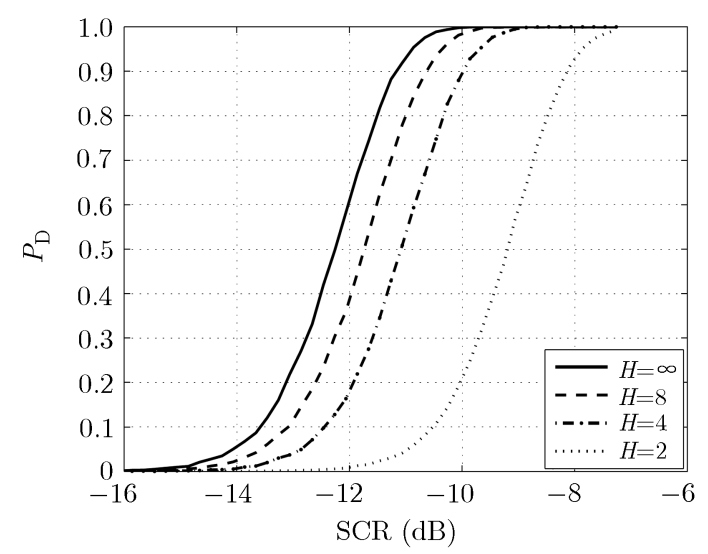

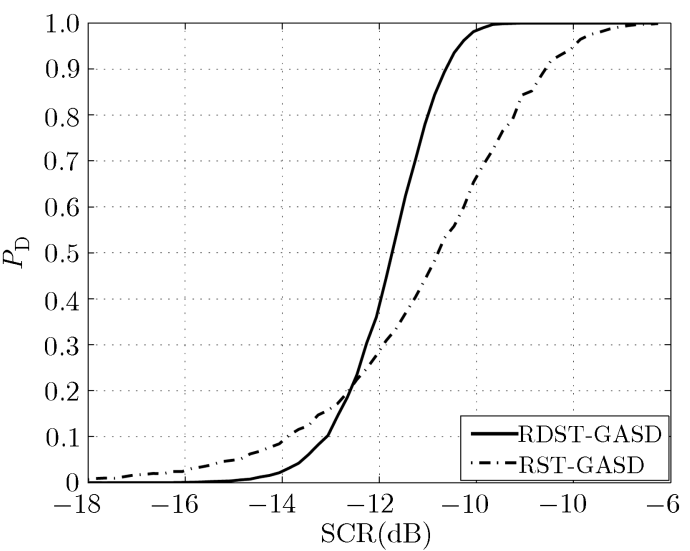

图 3 RDST-GASD 和 RST-GASD 检测性能比较

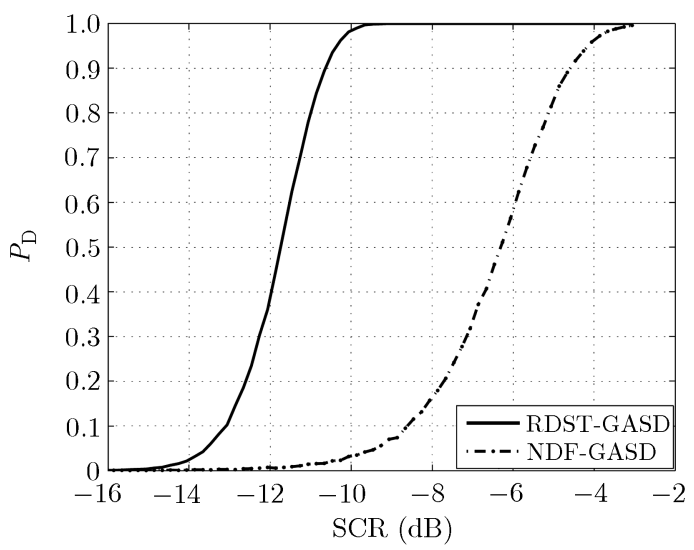

图 5 多普勒聚焦对宽带雷达检测性能的改善

图 4 参考单元长度不同时 RDST-GASD 的检测性能

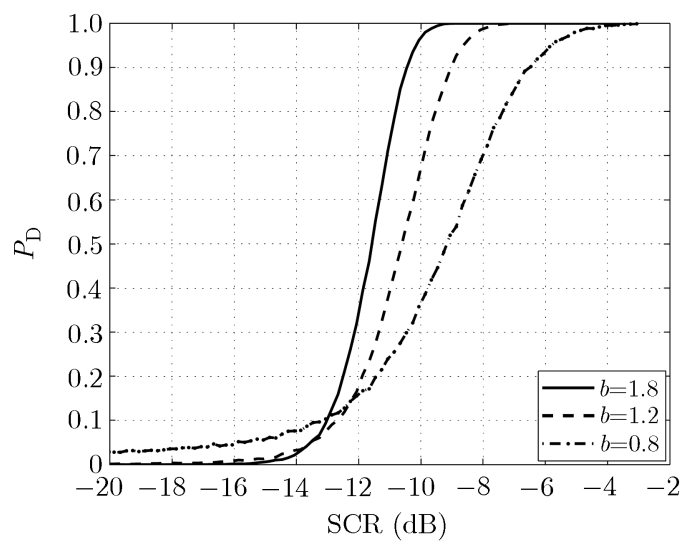

图 6 不同杂波参数下 RDST-GASD 的检测性能

Weibull 分布常用来作为高分辨雷达地面杂波的包络幅度的统计分布, 它也可以用球不变随向量 来表示, 其包络的概率密度函数为 $f_{r}(r)=a b r^{b-1} \exp \left(-a r^{b}\right)$, 其中 $r=\sqrt{\varepsilon}, a$ 是分布的尺度参数. $b$ 是分布的形状参数, $b$ 的取值越小, 杂波的方差就越大, 分布的拖尾越长, 杂波尖峰出现的概率也就越 高. 图 6 给出了在 3 种不同分布形状参数下 RDST-GASD 的检测性能, 随着杂波形状参数的减小, RDST-GASD 检测性能随之下降. 


\section{5 结论}

本文提出了一种用于高分辨雷达的非均匀杂波中的距离 - 多普勒扩展目标广义自适应子空间检 测器 RDST-GASD. 用距离频率一慢时间域的子空间模型描述在相参处理时间内存在越距离单元走动 的距离一多普勒扩展目标. 杂波模型用 SIRV 来描述. 将通过参考单元数据估计杂波的协方差矩阵用 于检测器中的杂波白化. 通过对检测统计量做统计分解证明了 RDST-GASD 具有恒虚警的性质. 检 测器利用 chirp-z 变换校正了目标回波的距离走动, 实现了相参积累, 从而改善了检测性能. 最后, 通 过一组 Monte-Carlo 仿真实验, 验证了 RDST-GASD 对非均匀杂波中的存在越距离单元走动的距离 一多普勒扩展目标检测的有效性. 对本文的研究工作可以得出以下几点结论: 1) RDST-GASD 符合距 离一多普勒双扩展目标的回波模型, 较之与模型失配的距离扩展目标检测器有一定的性能改善; 2) 距 离域中的非均匀杂波在距离频域变为均匀杂波, 从而简化了检测器的设计和计算; 3) 对于高分辨雷达, 做目标检测时必须考虑目标回波的越距离单元走动, 否则将存在严重的性能损失.

由于目标在相参处理时间内存在越距离单元走动, 因此检测窗的长度必须大于目标回波的支撑区, 导致积累损失增加 ${ }^{[14]}$. 尤其是当目标径向速度较大时, 积累损失将导致检测性能明显下降, 因此需要 将子空间检测器的输出在积累前变换回距离域, 并将积累窗收缩至与目标回波支撑区相当, 以减小积 累损失. 对这点的详细分析将在进一步的工作中发表.

\section{参考文献}

1 Conte E, de Maio A, Ricci G. GLRT-based adaptive detection algorithms for range-Spread targets. IEEE Trans Signal Process, 2001, 49: 1336-1348

2 Francesco B, de Maio A, Stefano A, et al. Adaptive radar detection of distributed targets in homogenous and partially homogeneous noise plus subspace interference. IEEE Trans Signal Process, 2007, 55: 1223-1237

3 Conte E, de Maio A, Ricci G. CFAR detection of distributed targets in non-Gaussian disturbance. IEEE Trans Aeros Electr Syst, 2002, 38: 612-621

4 Gerlach K, Steiner M J. Spatially distributed target detection in non-Gaussian clutter. IEEE Trans Aerosp Electr Syst, 1999, 35: 926-934

5 Chen V C, Li F Y, Ho S S, et al. Micro-Doppler effect in radar: phenomenon, model, and simulation study. IEEE Trans Aerosp Electr Syst, 2006, 42: 2-21

6 van Tress H L. Detection, Estimation, and Modulation Theory: Part III. New York: John Wiley and Sons, 2001. 413-443

7 Nicolas B, Khencharf A, Garello R. GLRT subspace detection for range and doppler distributed targets. IEEE Trans Aerosp Electr Syst, 2008, 44: 678-696

8 Rangaswamy M, Weiner D, Ozturk A. Non-Gaussian random vector identification using spherically invariant random process. IEEE Trans Aerosp Electr Syst, 1993, 29: 111-124

9 Kraut S, Louis L, Scharf L, et al. Adaptive subspace detection. IEEE Trans Signal Process, 2001, 49: 1-16

10 Conte E, Lops M, Ricci G. Adaptive detection schemes in compound-Gaussian clutter. IEEE Trans Aerosp Electr Syst, 1998, 34: 1058-1069

11 Gray R M. Toeplitz and circulant matrices: a review. Stanford 94305. Stanford: Department of Electrical Engineering, Stanford University, 2006. 32-34

12 Proakis J G, Manolakis D G. Digital Signal Processing, Principles, Algorithms, and Applications. New Jersey: Prentice Hall PTR, 1996. 482-486

13 Steven M K. Fundamentals of Statistical Signal Processing Volume II: Detection Theory. New Jersey: Prentice Hall PTR, 1998. 279-284

14 Orlenko V M, Shirman Y D. Non-coherent integration losses of wideband target detection. In: the 1st European Radar Conference. Amsterdam, 2004. 225-228 